\title{
PROGRAM ABH:
}

THE MODIFIED ABH METHOD OF CELL HOMOGENIZATION FOR TEMPEST

D. R. Skeen

JULY 1967

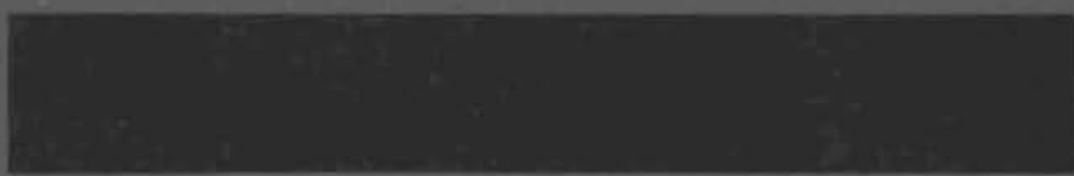

AEC RESEARCH \&

\section{DEVELOPMENT REPORT}

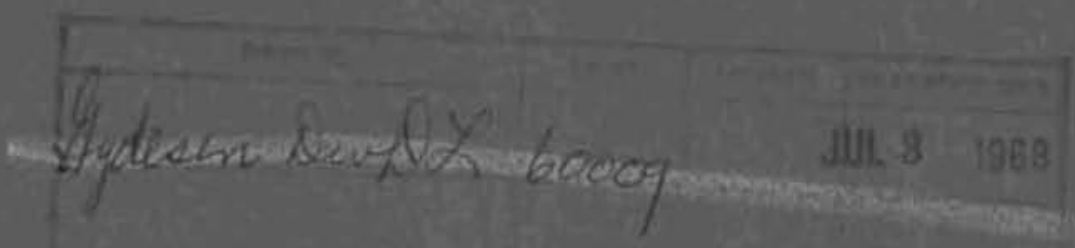




\section{LEGAL NOTICE}

This report was prepared as an uccount of Government sponsored work. Neither the. United States; nor the Commission, nor any person acting on behalf of the Commission:

A. Makes any warranfy or representation, expressed or implied, with respect to the accuracy, somplefeness, or usefulness of the information contained in this feport, or that the use of any information, opparatus, method, or process disclosed in this report may not infringe privately awned rights; or

B. Assumes any llabilities with respect to the use of, or for damages resulting from the use of any information, apparatus, method, or process disclased in this report.

As used in the above, "person acting on behalf of the Commission" includes any employee or contractor of the Commission, or emplayee of such contractor, to the extent that such employee or contractor of the Commission, or emplayee of such contracior prepares, disseminates, or provides access to, any information pursuant to his employment or contract with the Commission, or his employment with such cantracior.

PACIFIC NORTHWEST LABORATORY

RICHLAND. WASHINGTON

operated by

BATTELLE MEMORIAL. INSTITUTE

for the

UNITED STATES ATOMIC ENERGY COMMISSION UNDER CONTRACT AT(45-1)-1830 


$$
\begin{gathered}
\text { BNWL- } 515 \\
\text { UC-80 Reactor Technology } \\
\text { Special Distribution }
\end{gathered}
$$

PROGRAM ABH:

THE MODIFIED ABH METHOD OF CELL HOMOGENIZATION FOR TEMPEST

By

D. R. Skeen

Theoretical Physics Section

Reactor Physics Department

July 1967

FET ID:

Divisis

PACIFIC NORTHWEST LABORATORY

RICHIAND, WASHINGTON 
I. INTRODUCTION ................

II. THEORY OF THE MODIFIED ABH METHOD. . . . . . .

III. INPUT DESCRIPTION FOR PROGRAM ABH. . . . . . . 5

IV. $\mathrm{ABH}$ PROGRAM IISTING. . . . . . . . . . . . . 8

V. ABH AND TEMPEST SAMPLE CASES . . . . . . . . 25 


\section{SECTION I: INTRODUCTION}

The disadvantage factor theory, as originally presented by A. Amouyal, P. Benoist and J. Horowitz, $(1,2)$ has been programmed, with some modifications, in FORTRAN IV for the Univac $1107-8$ computer. The program is intended to be used in conjunction with program TEMPEST $(3)$ to produce cell cross sections for use in broad-group constants codes.

The modifications to the Amouyal, Benoist and Horowitz theory are principally those described by Strawbridge, (4) which allow for the use of cladaing, instead of a void region, surrounding the fuel. In addition, a minor correction was introduced by the author and $A$. G. Gibbs which improves the treatment of the current relationships at the fuel and clad boundaries (Section II A). The entire theory with its modifications is described in section II.

Input for program ABH, which is described in Section III, is via MAMEITI. Basically this code takes a set of cross sections from an existing TEMPEST library tape and calculates, for each TEMPEST energy, a set of disadvantage factors for a three region (fuel-clad-moderator) cell. The crosis sections ar: then multiplied by these factors and a new TEMPEST library tape is written. Having thus smeared the cell spatially, the energy averaging may then be done by running a smeared cell case with program TEMPEST. Although the major" portion of the program is new, mention should be made of the TEAM-AMBER $(6,7)$ code from which certain sections of logic were lifted. 
SECTION II: THEORY OF THE MODIFIED ABH METHOD

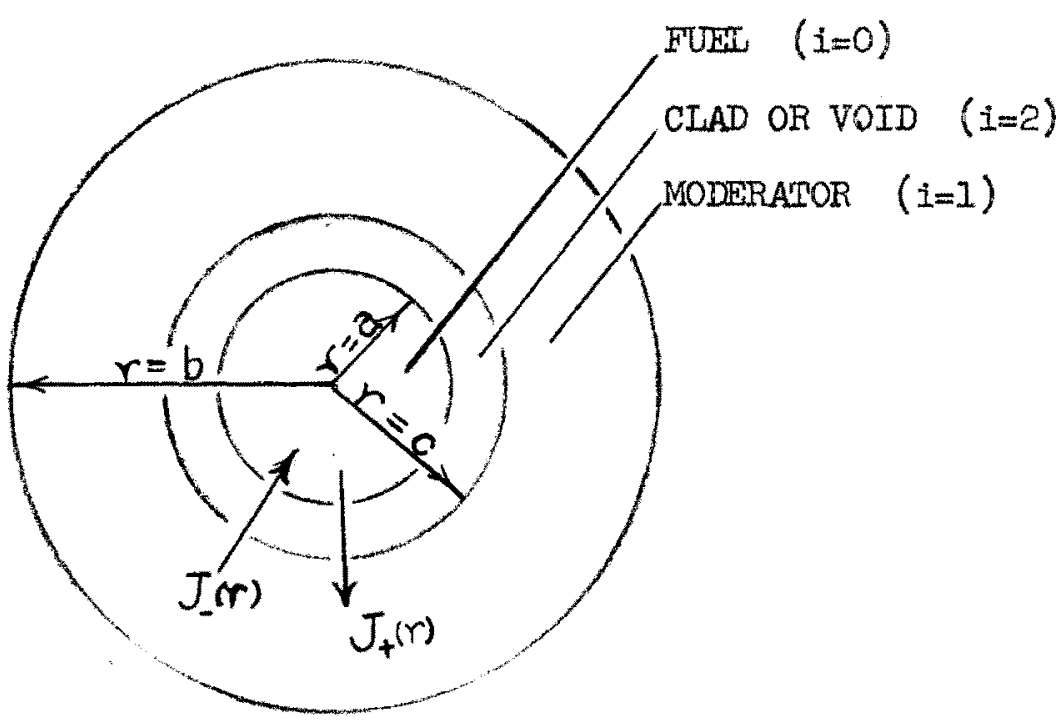

Figure 1 The Modified ABH Cell

Assume the following nomenclature,

$F_{i}=$ The volume fraction in the $i^{\text {th }}$ region of the cell, whose volume is $V_{1}$. Radii and region numbers are shown in Figure $1_{3}$

$\phi_{i}=$ The average $f$ lux in the $i^{\text {th }}$ regaion of the cell which contains a void instead of cladding,

$\hat{\phi}_{i}=$ The average flux, corrected for cladding, in the $i^{\text {th }}$ region of the cell,

and

$\langle\phi\rangle=$ The average flux in the whole cell. 
The following equations are used to calculate disadvantage factors by the modified ABH method.

$$
\frac{\phi_{1}}{\phi_{0}}=\frac{\mathrm{a}^{2} \mathrm{~A}}{\left(\mathrm{~b}^{2}-\mathrm{c}^{2}\right)} \quad \frac{{ }^{\Sigma} c_{0}}{\sum_{c_{1}}}-\frac{2 \mathrm{a}^{2}}{\mathrm{c}} \Sigma_{c_{0}}+\frac{\mathrm{a}}{\mathrm{c}} \frac{1}{(1-\mathrm{B}) \mathrm{P}}
$$

where

$$
A=3 \Sigma_{t r} \Sigma_{c_{1}} b^{2} \hat{c}+\frac{3}{2} \frac{V_{1}}{c} \Sigma_{c_{1}} \lambda
$$

and

$$
B=\frac{2 \sqrt{2}}{\pi} \quad \frac{c-a}{c}
$$

$\lambda$ is tabulated as a function of $\Sigma_{t_{1}}$ and $\hat{C}$ is calculated as

$$
\hat{c}=\frac{1}{2}\left[\ln \left(\frac{b}{c}\right) \frac{b^{2}}{b^{2}-c^{2}}-\frac{3}{4}+\frac{1}{4} \frac{c^{2}}{b^{2}}\right]
$$

$$
\frac{\hat{\phi}_{1}}{\phi_{0}}=\frac{\phi_{1}}{\phi_{0}}+\mathrm{a}(\mathrm{c}-\mathrm{a}) \Sigma_{\mathrm{c}_{0}}\left[\frac{3}{2} \Sigma_{t r_{2}}-\frac{1}{\mathrm{c}}\right]+\frac{1}{2 \mathrm{P}}\left[\frac{(\mathrm{c}-\mathrm{a})}{\mathrm{c}}-\frac{2 \mathrm{~B}}{1-\mathrm{B}}\right]
$$

$$
\frac{\hat{\phi}_{2}}{\phi_{0}}=\frac{1}{\mathrm{P}}-a \Sigma_{\mathrm{c}_{0}}+\frac{3}{4} \quad(\mathrm{c}-\mathrm{a}) \mathrm{a}{ }_{\operatorname{tr}}{ }{ }^{\Sigma} \mathrm{c}_{0}
$$

$$
\frac{\langle\phi\rangle}{\phi_{0}}=F_{0}+F_{2} \frac{\hat{\phi}_{2}}{\phi_{0}}+F_{1} \frac{\hat{\phi}_{1}}{\phi_{0}}
$$

$$
\frac{\hat{\phi}_{2}}{\langle\phi\rangle}=\frac{\hat{\phi}_{2}}{\phi_{0}} \cdot \frac{\phi_{0}}{\langle\phi\rangle}
$$

$$
\frac{\hat{\phi}_{1}}{\langle\phi\rangle}=\frac{\hat{\phi}_{1}}{\phi_{0}} \cdot \frac{\phi_{0}}{\langle\phi\rangle}
$$


where $\mathrm{P}$ can be calculated by a method suggested by Amouyl, Benoist, and Horowitz ${ }^{(1)}$

$$
P=\frac{1}{1+\frac{{ }^{c_{0}}}{\Sigma_{T_{0}}}\left[x\left(1+\alpha \frac{{ }^{2} s_{0}}{\Sigma_{T_{0}}}+\beta \frac{\Sigma_{\Sigma_{0}}}{\Sigma_{T_{0}}^{2}}\right)+a \Sigma_{T_{0}}\right]}
$$

where $x, \alpha$, and $\beta$ are tabulated as functions of $\Sigma_{t_{0}}$. 
SECTION III: INPUT DESCRIPTION FOR PROGRAM ABH

\begin{abstract}
All input to this program is via NAMELIST. (5) The following is a description of the variables which may be input via NAMEIIST. One note of warning, be sure to have a TEMPEST library tape listing handy when preparing input for Program ABH.
\end{abstract}

A. \$ Input

Variable

ISO $(I, J, K)$

$\operatorname{DEN}(I, J, K)$

$\operatorname{RAD}(K)$

NUM(K)

OUT

\section{Definition}

Isotope ident numbers from the TEMPEST library tape to be used in the $\mathrm{ABH}$ calculation and whose values will be subsequently modified for the new TEMPEST tape.

Isotope region densities to correspond to the isotope numbers given above.

Region radii in $\mathrm{cm}$.

The number of isotopes in each region.

Output control (INTEGER); OUT $=0$, short, OUT $=2$, will list new tape and the disadvantage factors.

The above indices are defined as:

\begin{tabular}{|c|c|c|c|c|}
\hline Index & \multicolumn{4}{|c|}{ Definition } \\
\hline$I$ & \multicolumn{4}{|c|}{$\begin{array}{l}\text { The index of the cross section in this region } \\
\text { (maximum of } 50 \text { ). }\end{array}$} \\
\hline \multirow[t]{2}{*}{$J$} & Normal TEMPEST & & QMPEST & $A B H$ \\
\hline & Ident. Multiplier & $\mathrm{RS}(5)$ & Cross Section & Usage \\
\hline 1 & 0 & -- & Absorption & Absorptic \\
\hline 2 & 100 & $\nu$ & Fission & $-\infty$ \\
\hline 3 & 200 & $1-\bar{\mu}$ & Scatter & $(1-\mu) \sigma_{c}$ \\
\hline 4 & 300 & $\xi$ & Scatter & $\sigma_{s}(o n i y)$ \\
\hline $\mathrm{K}$ & \multicolumn{4}{|c|}{$\mathrm{ABH}$ region radii in $\mathrm{cm}$. } \\
\hline 1 & \multicolumn{4}{|c|}{ Fuel radius } \\
\hline 2 & \multirow{2}{*}{\multicolumn{4}{|c|}{$\begin{array}{l}\text { Cladding outer radius } \\
\text { Moderator outer radius }\end{array}$}} \\
\hline 3 & & & & \\
\hline
\end{tabular}


Note that this section of input is set up so that each cross section ident can be input. Thus, for the same material, one can use different scattering; and absorption cross sections (not just those which differ by 100 multiples). This was necessitated by the fact that the present TEMPEST library will, for some isotopes, store 1.0 for $\xi$ ( in the RS(5) position) and then store $\xi \sigma_{S}(E)$ for $\sigma_{S}(E)$ making it impossible for $A B H$ to pick up the proper scatter cross section. Normally, however, the only number that need be input is the base library ident. $[1 \leq \mathrm{ISO}(I, J=I, K) \leq 99]$ and multiples of 100 will be assumed for the other cross sections.

Following the make up of the cell disadvantage factors there is one final stage of input so that any final shifting can be done prior to output on the new tape.

B. \$ EDIT

$\underline{\text { Variable }}$ $\operatorname{ISO}(I, J, K)$

$\operatorname{RSLAST}(I, J, K)$
Definition

For input of cross section idents to cheng: the cross sections being output on the new TEMPEST tape.

Anything entered here will be placed in the last position of the RS vector before beting output on the new TEMPEST library tape.

A description of the TEMPEST library tape will help describe the RSLAST input. The TEMPEST library tape format is as follows:

NAME IDENT NO. NO. RT VALUES

\begin{tabular}{|lllll|}
\hline & & & & \\
\hline $\mathrm{E}^{\sigma_{2200}}$ & 2 & 3 & 4 & 5 \\
\hline $\mathrm{RS}$ & & & & \\
\hline 1 & 2 & 3 & 4 & $\mathrm{RS}(5)$ \\
\hline
\end{tabular}

$\mathrm{RT}$ Tabulated cross sections if No. RT values $\neq 0$. Not present if No. Rt. Values $=0$.

For a discussion of those parameters see program TEMPEST 
For cross section idents of 100 through 300 the $\mathrm{RS}(5)$ is used for storage as shown under \$INPUT. It is this RS(5) position which is loaded when the new library tape is written. For example, in the test case, it became necessary to use the 431 hydrogen scattering cross section. However this isotope does not have $\xi$ stored in $\mathrm{RS}(5)$ so that $\xi_{\mathrm{S}}(\mathrm{E})$ could not be properly supplied for TEMPEST. Therefore $\xi$ was input in the test case as shown in Appendix A. 
$-8-$

BNWL -515

SECTION IV

ABH PROGRAM IISTING 


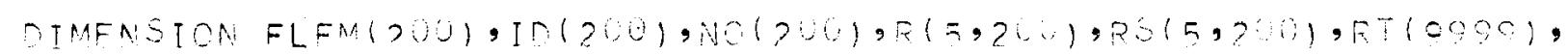

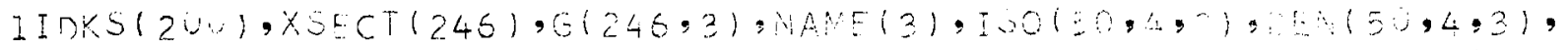

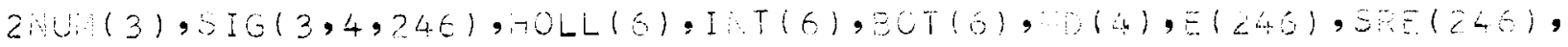
3ROUT (5), RSOUT (5), RSLAST $(5,4,3), S$ SP (6)

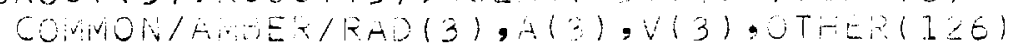

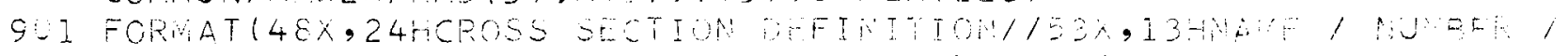
$156 \times$ 7HLENSITY / $53 X$ I3ASEAR GNSITY

2 HISH AOSORPTION
3ATTER

L. 7104 -

a.

QU2 FOFMAT(E)(4X,A6,2H /,I $4,4 x) / 2(6(4 x, 3 P E] 1,5,5 x) / 1)$

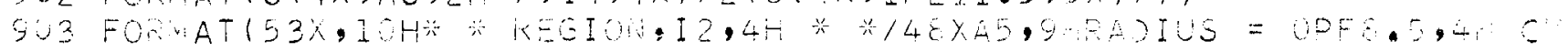

94 FOART(8) I $4,1 P E 11.4)$

QUS FORWAT(26AFLUX OEPRESSIOA FACTORS (, A5,2H))

Q.6 FORAT $(3(4 X, 46,2 H /, I 4,4 x) / 2(3(4 x, 2 P(11.5,5 x) / 1)$

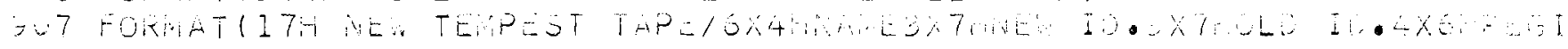

IUNTX13HGLL THPE LCC.43XITHO. ERECY POTATSI

G.S FORXAT(5XAG,I7, 2IIU, IIS, $57 \times$ I5)

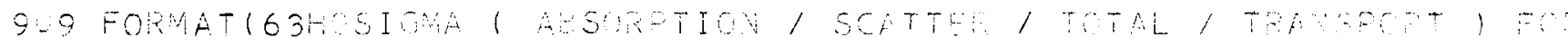
$1 F-6 T C A 13)$

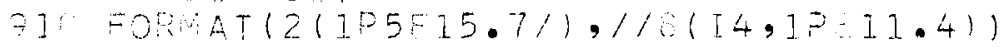

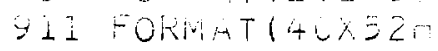

$123 \times 52 \mathrm{H}$

$333 \times 581$

$133 \times=94$

$333 \times 29-1$

$323 \times 50 \mathrm{H}$

$\therefore 23 \times 50 \mathrm{H}$

$522 \times 504$

$62 \times 2 \times \mathrm{OH}$

$722 \times 0 \Omega H$

$2=2 \times 504$

$022 \times 594$

$33 \times 595$

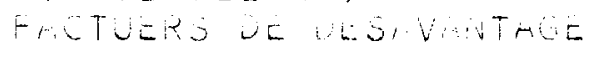

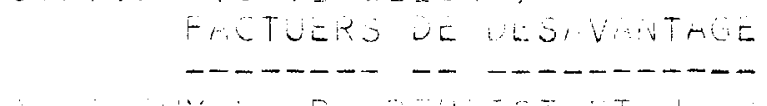

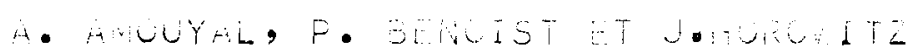

minas

$332 \times 5014$.

$32 \times 5971 \%$

$x 3 \cap y$

4625

$\times 30 \times$

$5.62+1$

12 FOXTATIOCX

$66 ?+1$

$x \sin x$

$76 ? \mathrm{P}$

$\times 30 \times$

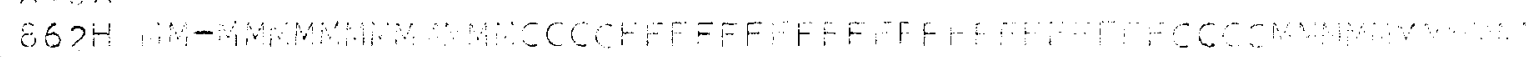


$x 30 x$

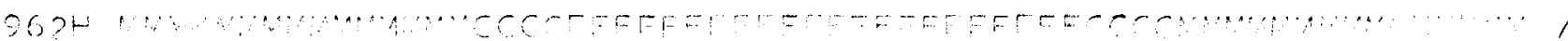

$x 30 x$

$1621: 1$

$x 3, x$

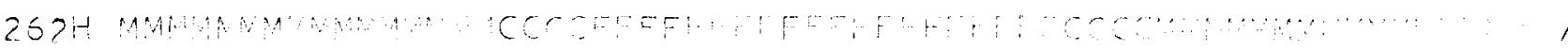

$x \geqslant 0 \times$

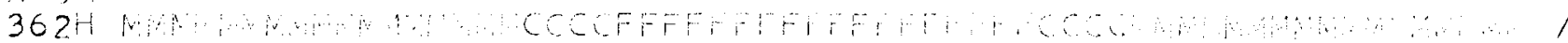
$x 2 n x$

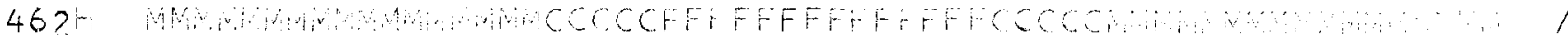

$\times 30 \times$

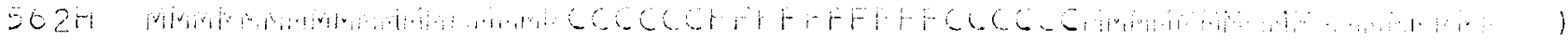

913 TORMATI $: X$

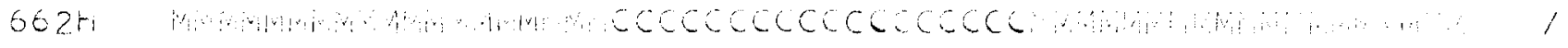

$\times 30 \times$

7525

$\times 21 \times$

$8 \in 2 H$

$x ? \cap x$

$962-$

$x 3 n x$

$1<04$

$222 \times 234$

$233 \times 234$

andmand acecectect.

$x=1$

$46 ?$

$\times 20 x$

5625

$622 \times 50 \mathrm{H}$

$733 \times 59-1$

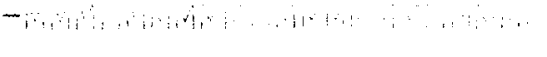

ino

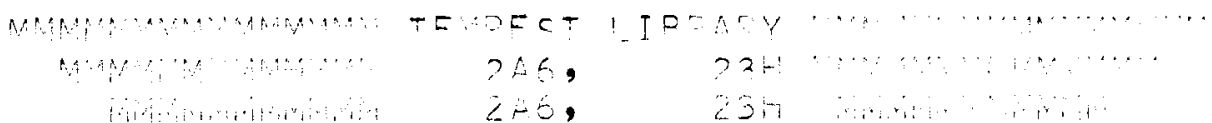

$2+6$,

235

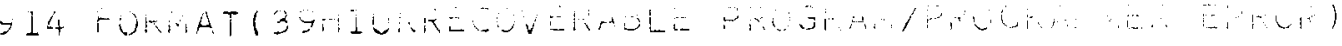

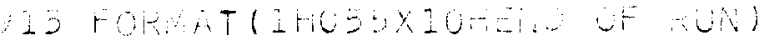

916 FOPHAT $(1 H)$

PATA(E(I), I= ?, 12?)/

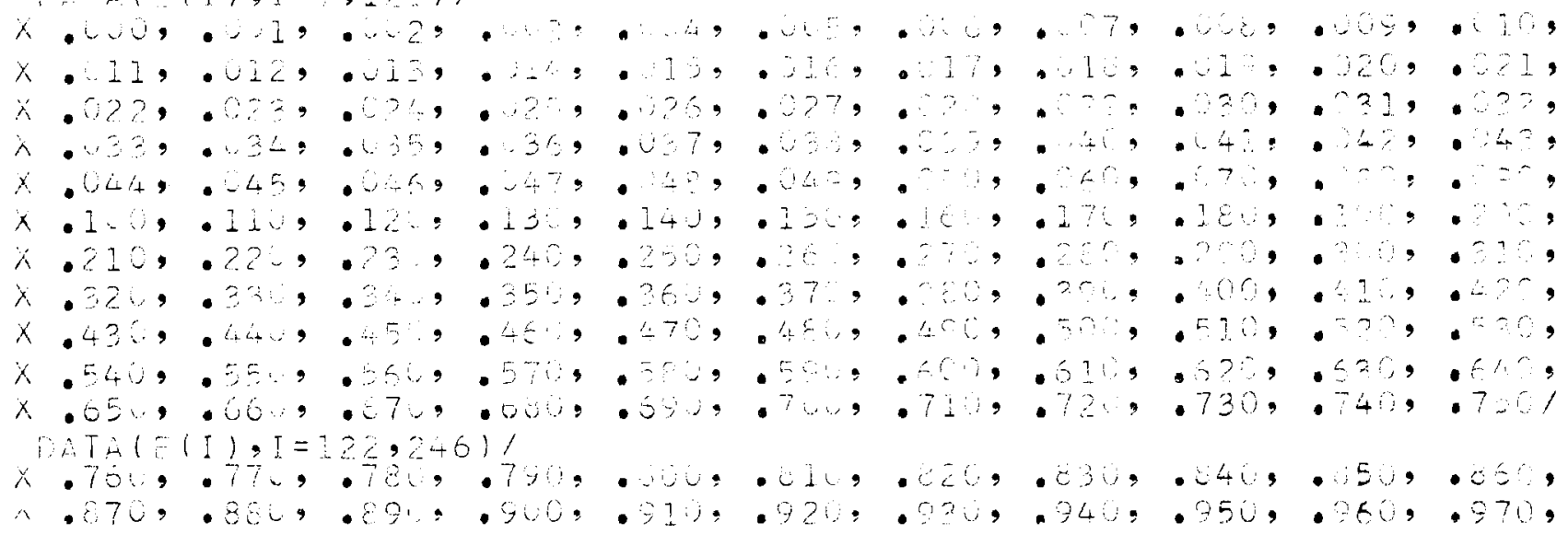




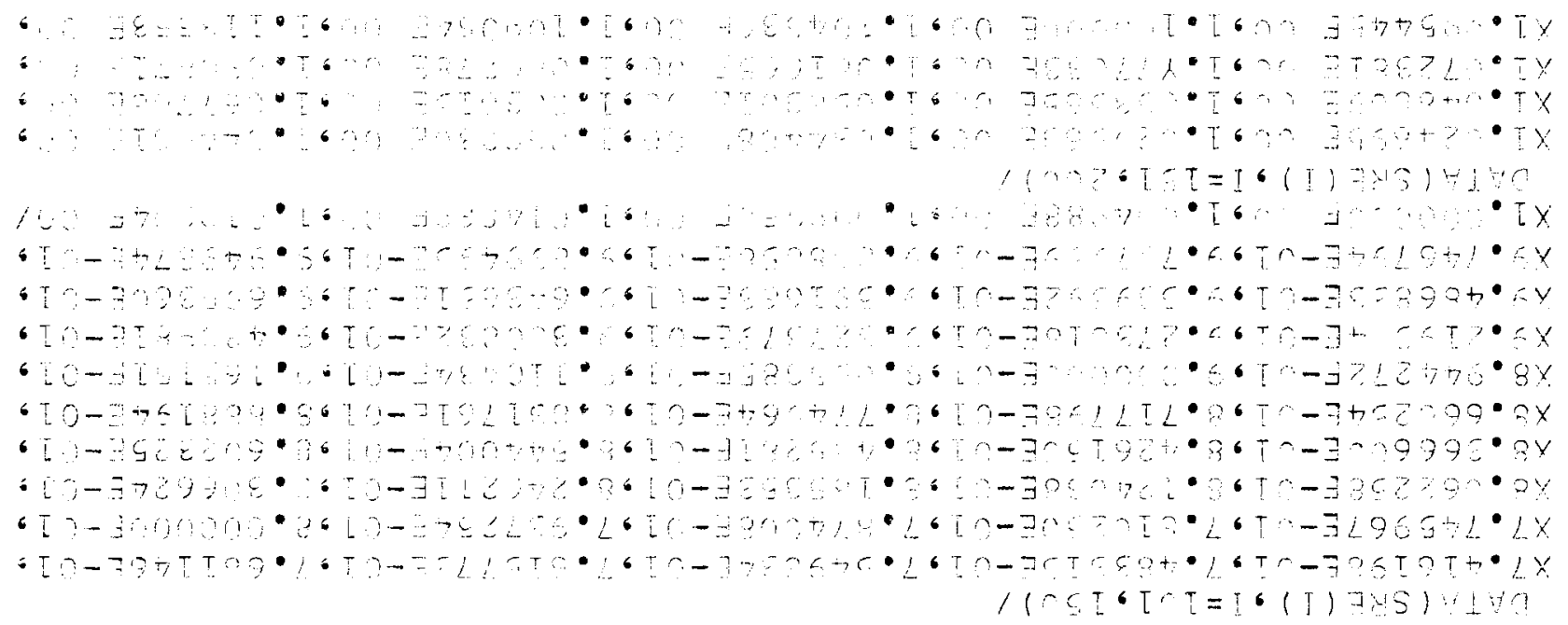

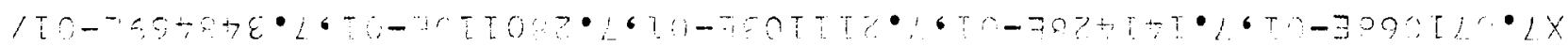

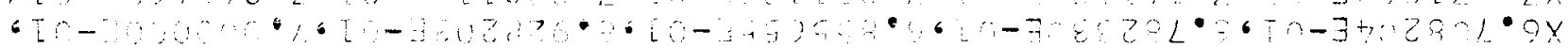
- 1 -

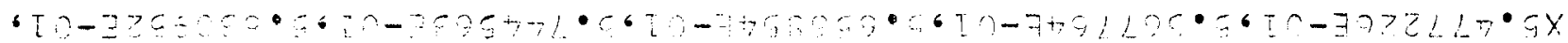

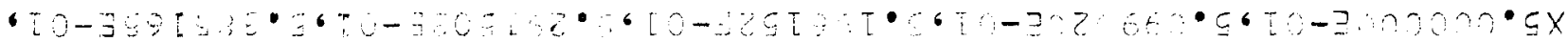

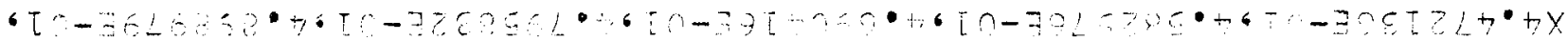

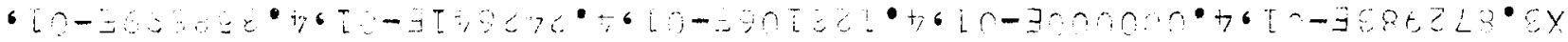

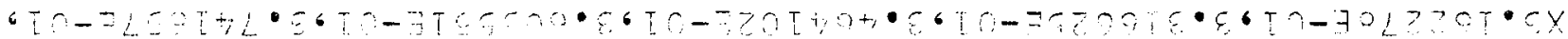

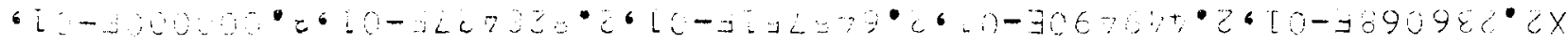

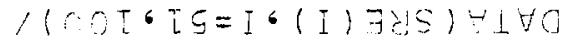

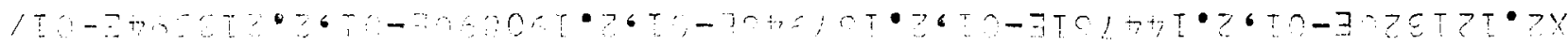

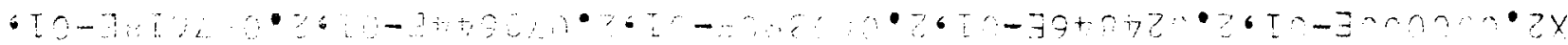

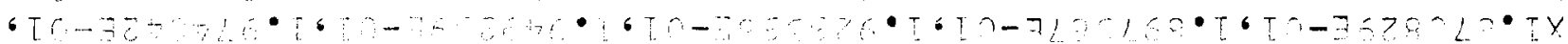

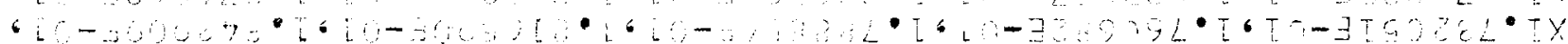

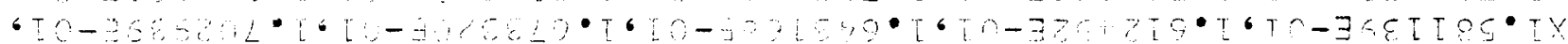

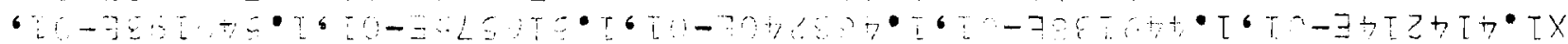

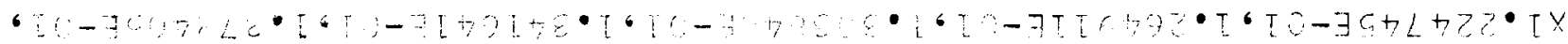

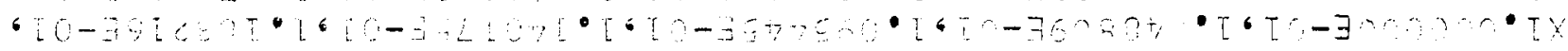

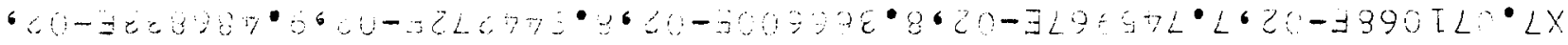

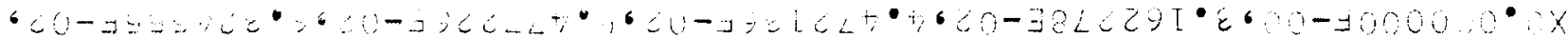

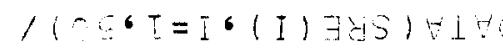

$100 \cdot 76 \div 6 \cdot 7 \cdot 796 \cdot T \cdot O L G \cdot T X$

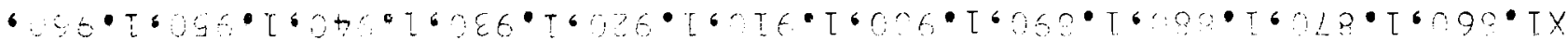

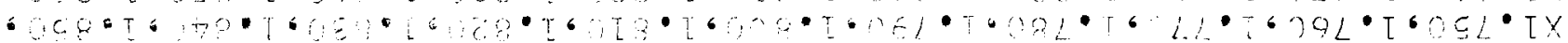
-

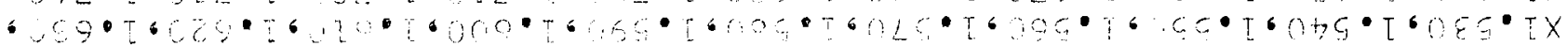

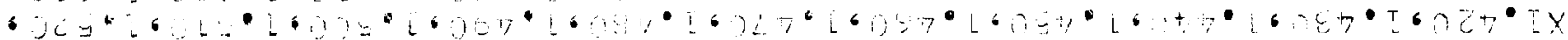

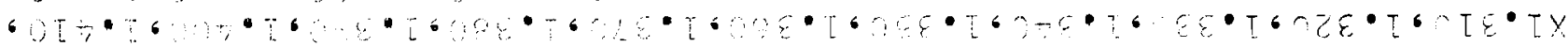
6 J

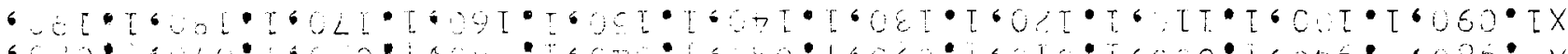

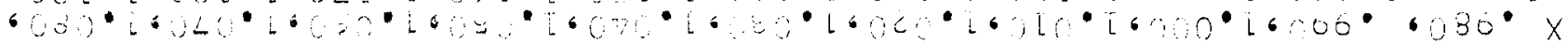




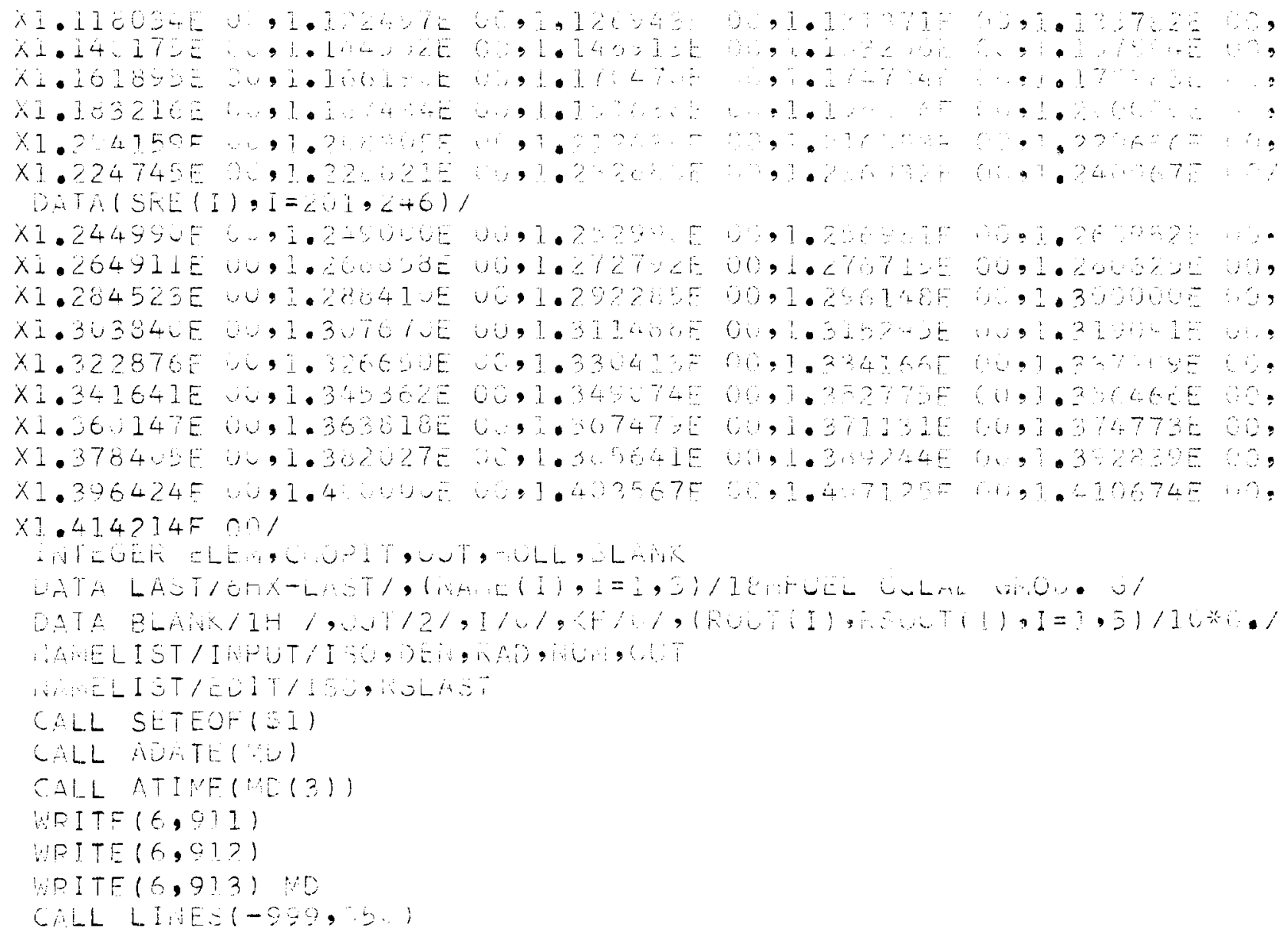

C 


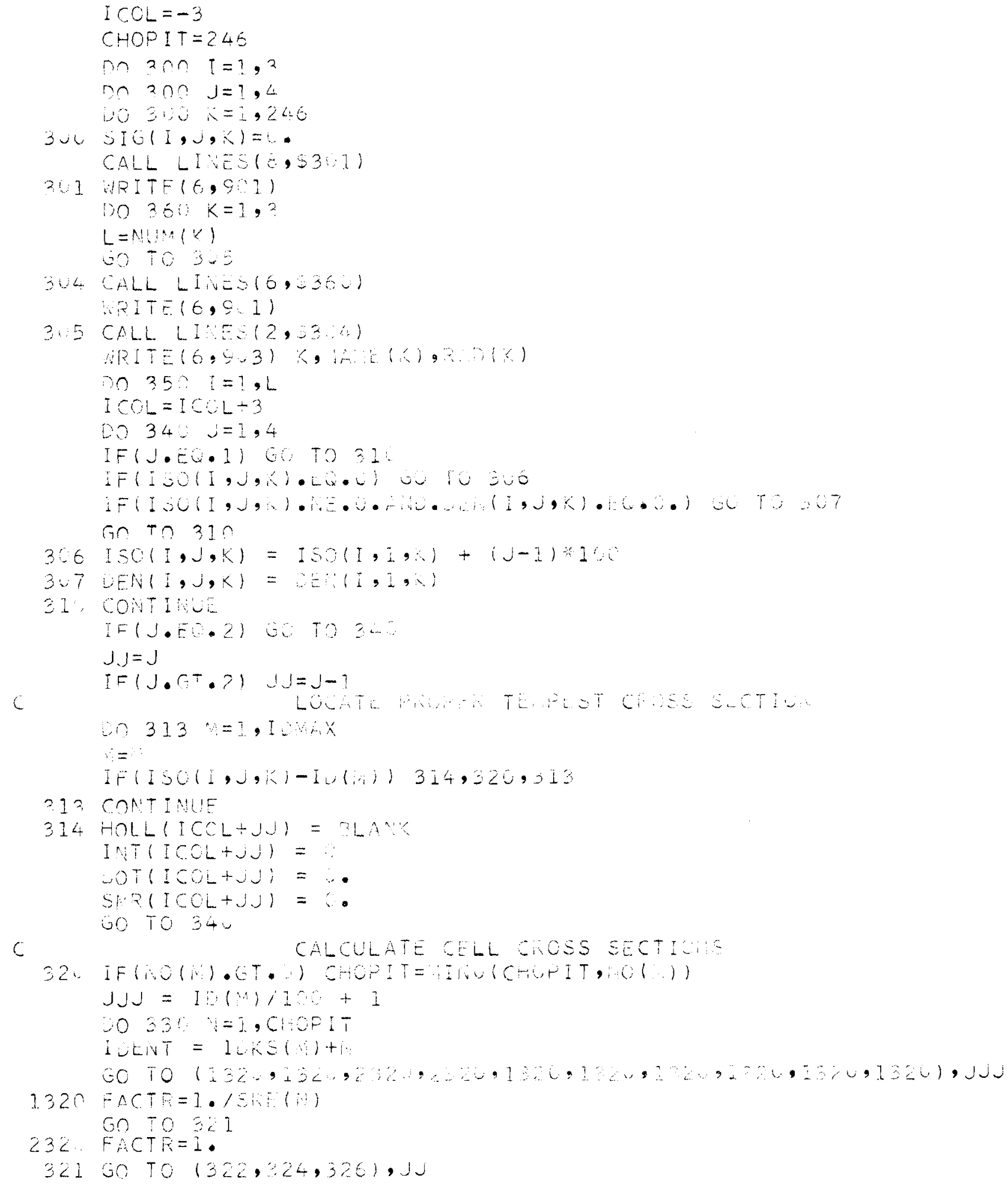




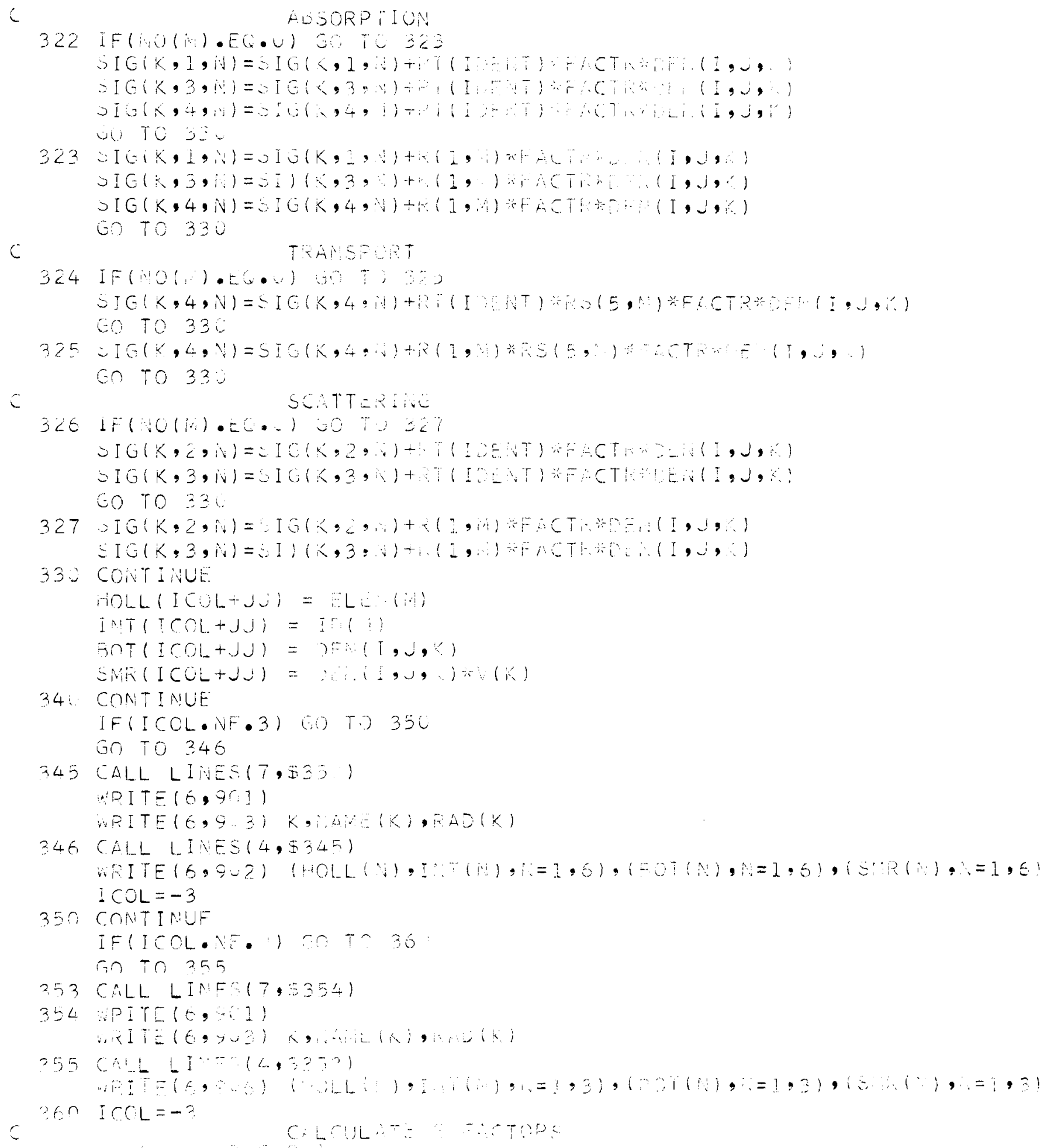




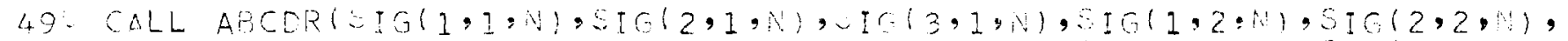
$1-1 G(2,2, N), \therefore I G(1,3, N), S I G(2,2, N), I G(2,2, N), E I C(1, L, N), S T C(2,4, n)$, $2+16(3,4,4), C(6,1), 0(A=2), 6(1,3))$

$(i, 1)=C(::)$

$(6(1,2)=6(2,2)$

$G(1,3)=6(2,3)$

$A S P=(6-9 P T+7) / 8$

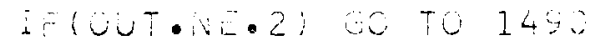

$00440 \% \quad k=1$ ? ?

CNLL LINES(NGP+4, 02496$)$

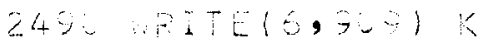

$00,449, j=1,4$

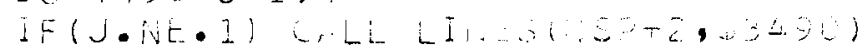

VITF $(6,016)$

$2 / 490$ ar $4 \angle 0, \quad I=?, \quad 3$

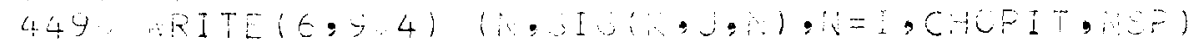

ILy $\quad 1 F(0, T \cdot L) I)$ Go TO $5 \ldots$

c

$0405 k=1$

CUP 1 के

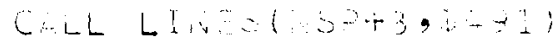

491 \%

$10<95 \quad i=i, 4<0$

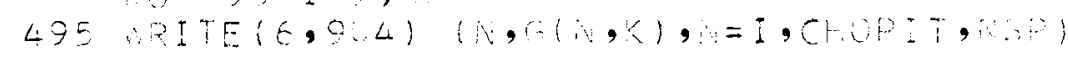

C

SKIFE MLO TEMPEST T,

$50 O$ PEAD (E,EOIT)

IF(OUT.ME.?) C.LL L I \%S1-1, \%5.:2)

GO TO $=?$

301 a स $75(6,07)$

$50200600 \mathrm{~J}=1,4$

F IO $=10+(j-1)$

Q $6<-\therefore=?$

$L=\sin (\mathrm{K})$

$000 \quad I=1$

$N=I D=(-1)+?$

iO $510 \cdots=1, i C A x$

$\therefore=\cdots$

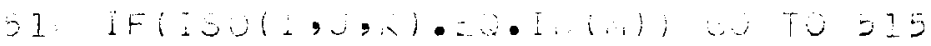

CO TO 6

$515 \mathrm{JJJ}=10(\mathrm{~d}) / 160+i$

LO $99, n=I, C O P I T$

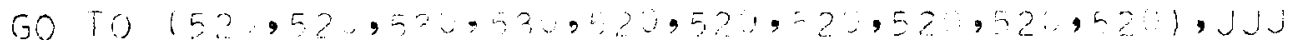

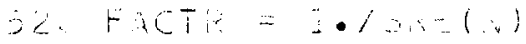

Gn Tr 54

5,$30 ;-T=\therefore$

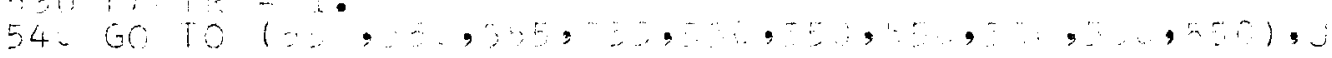

55. FACTR = FACARASRA(A) 


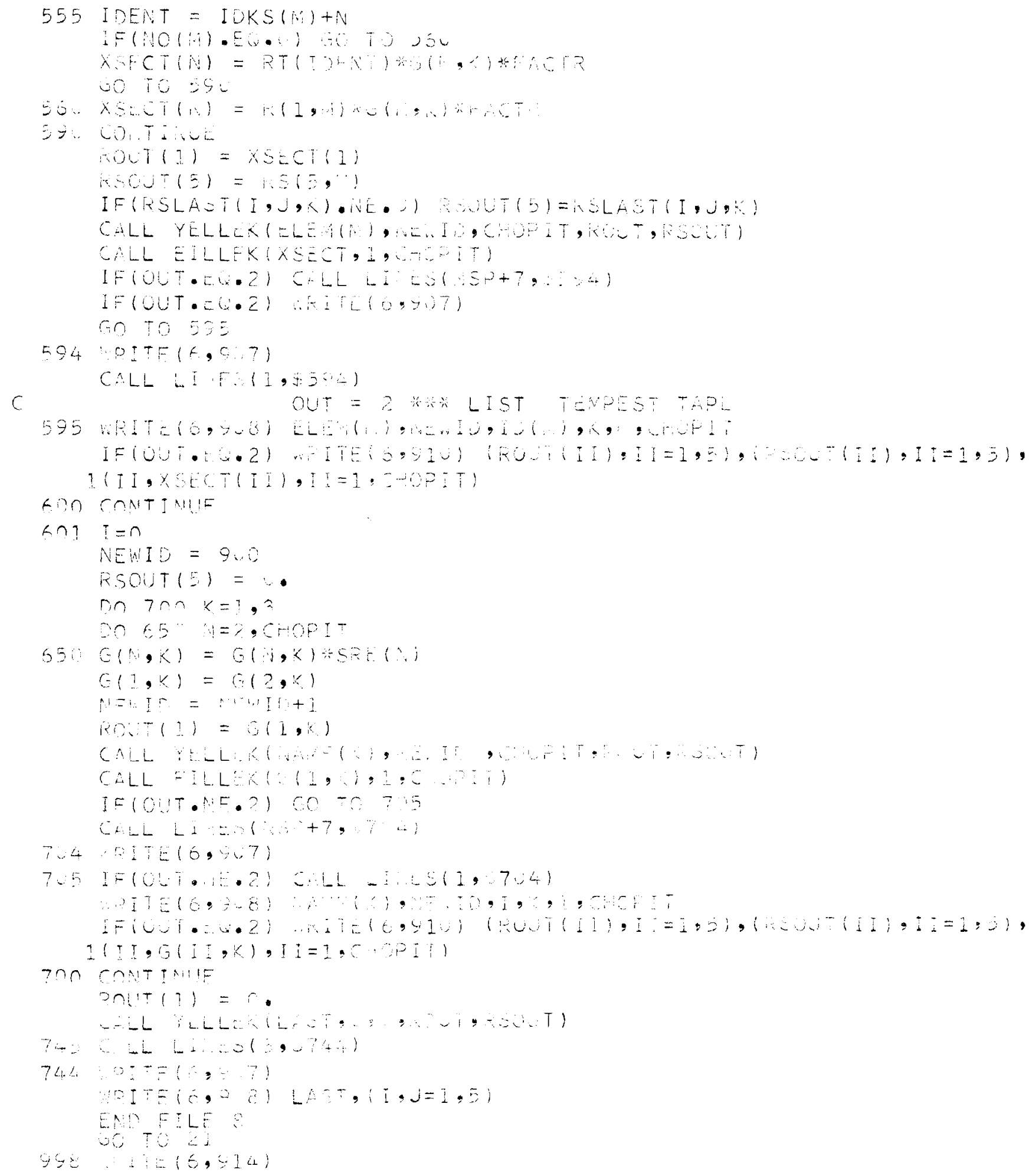




$$
\begin{aligned}
& \text { Gก Tก } 990 \\
& 1 \text { SRTE }(8) \text { W } \\
& \text { =MR FILF? } \\
& 99=a+n \\
& \text { FuL Exit }
\end{aligned}
$$




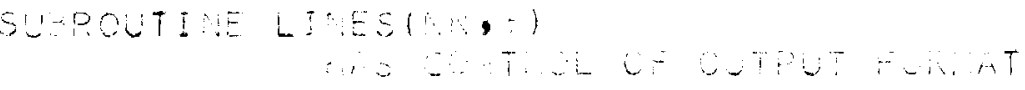

$$
\begin{aligned}
& \text { L } \\
& \because-\cdots+\cdots+\cdots
\end{aligned}
$$

$c$

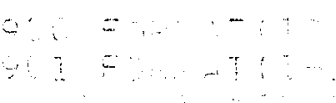

$$
\begin{aligned}
& +1+\infty, 1,1, \\
& 10 \div \div=2 f+ \\
& {[+;, \cdots, \cdots,} \\
& -T+\cdots=26
\end{aligned}
$$

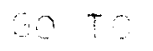

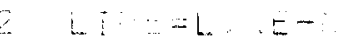

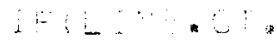

$$
\begin{aligned}
& \text { la }-i \quad z=-1
\end{aligned}
$$

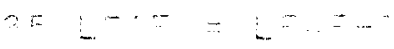

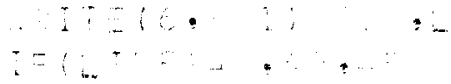

$$
\begin{aligned}
& 4 . \cdots=
\end{aligned}
$$$$
=T T+
$$$$
\text { , } 12+6 .
$$

$T: \quad T^{-} \quad T \quad \cdots \quad T$ $\because \cdots ;$ 


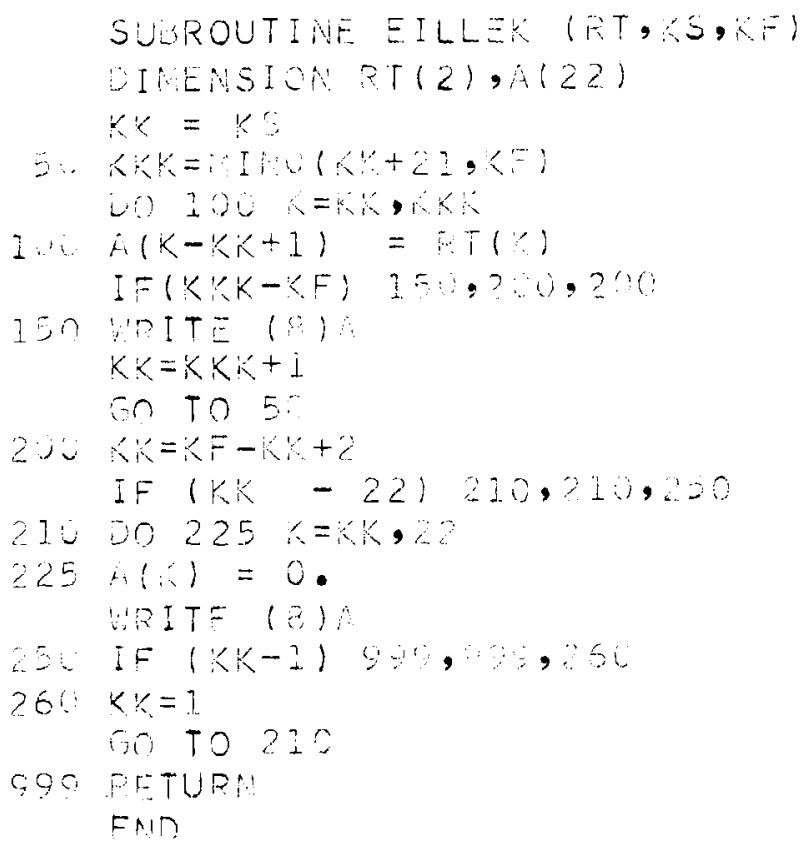




$$
\begin{aligned}
& \text { SUBROUTINE YELLEK (E, I, R, RS) } \\
& \text { CIUENSICN A }(22),(2), 9 S(2) \\
& A(1)=F \\
& \therefore(2)=\mathrm{I} \\
& \therefore(2)=N \\
& 0,1, \cdots=3,5 \\
& (A(x+3)=2(4) \\
& 110(x+2)=85(6) \\
& 10035: x=14,: ? \\
& 150 A(B)=0 \\
& \text { WRITE (e): } \\
& \text { RETUR: } \\
& \text { EN? }
\end{aligned}
$$




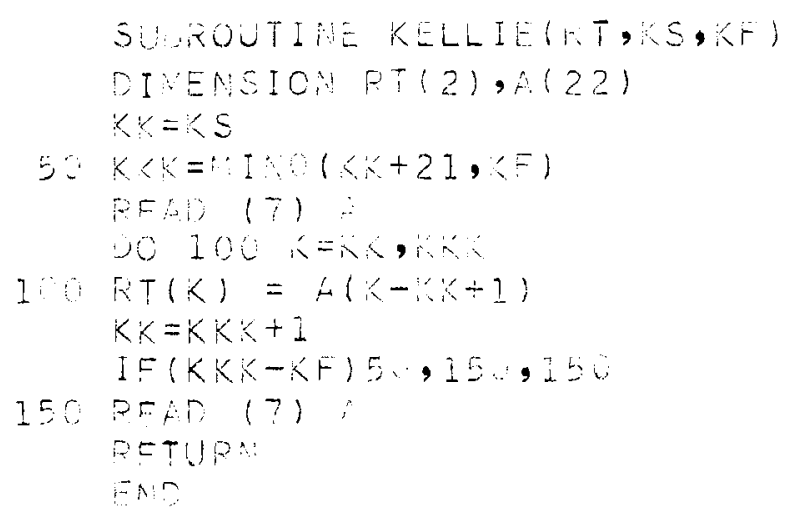




$$
\begin{aligned}
& \text { SUOROUTALE RELLEY(A, 1, Q, DS }
\end{aligned}
$$

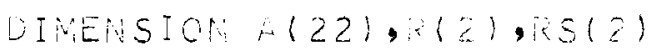

$$
\begin{aligned}
& \text { Pan (?) i } \\
& E=A(?) \\
& I=A(?) \\
& \because=(3) \\
& \text { On } 10 \mathrm{n}=, 5 \\
& R(k)=i(k+3) \\
& \text { I... K K }(K)=(K)+B) \\
& \text { DETIIR: } \\
& \text { Enin }
\end{aligned}
$$




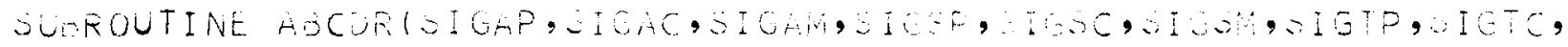

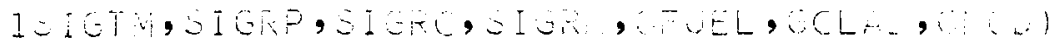


SUEROUTINE PRECON

COMMON/AMBER/S(3), A (3), V(3), T, , COTHEA(3),

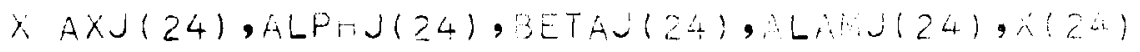

DATA(AXJ(B), $=7,24) /$

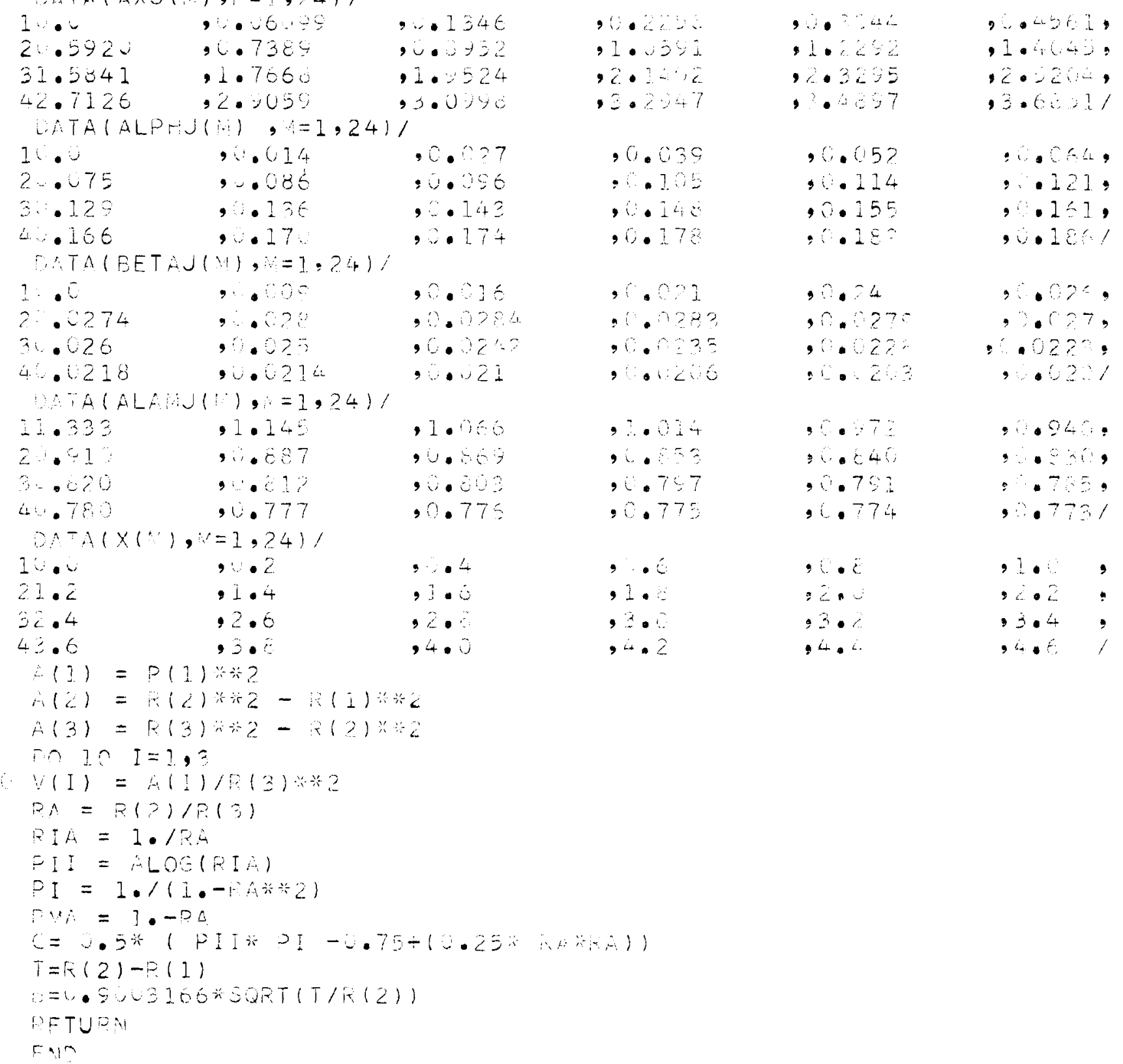


$=4-515$

$-25$

SECTION $V$

ABH AND TEMPEST SAIPLE CASTS 


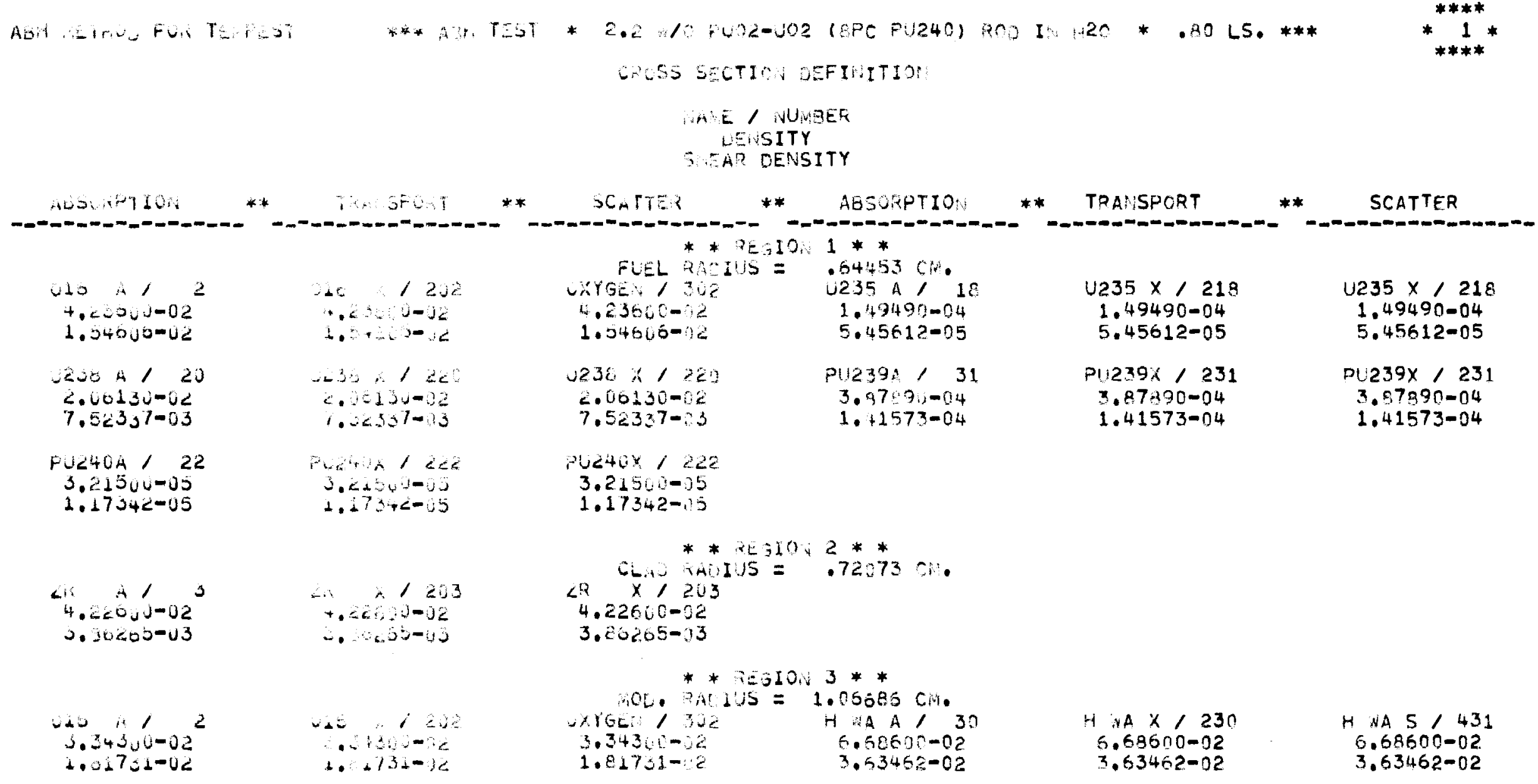




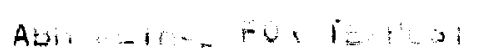

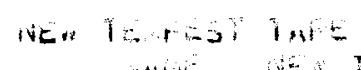

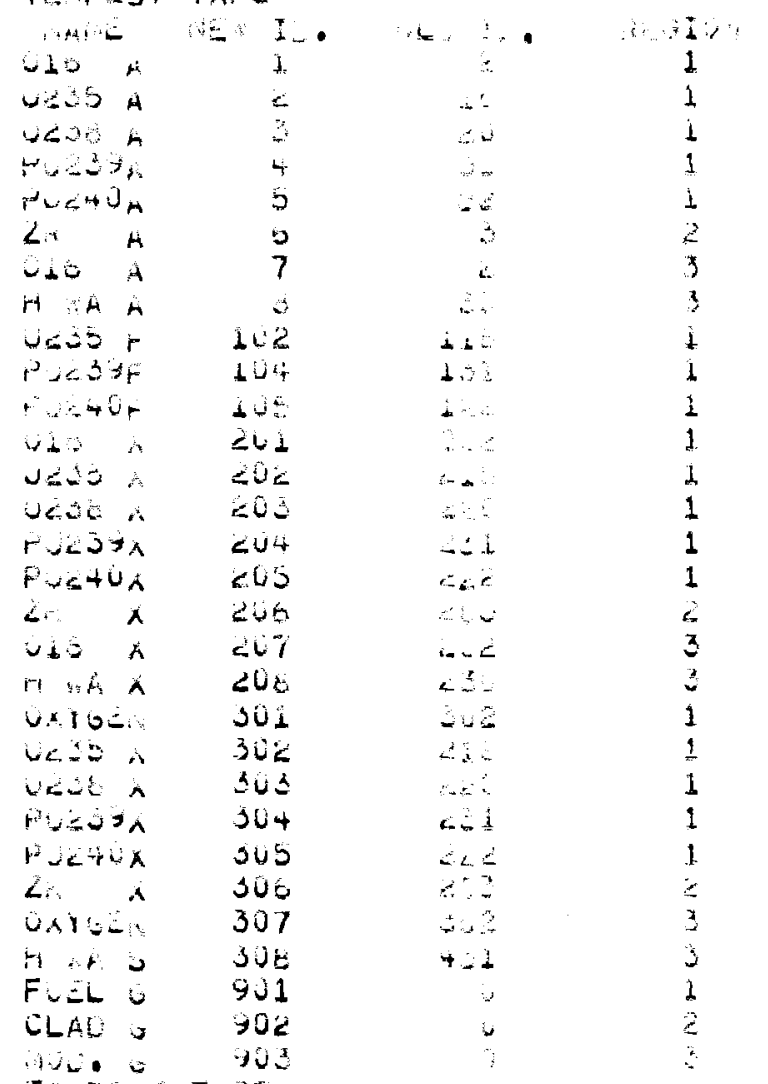

NE $T$ is THEST TAPE $\therefore \operatorname{Min}$ OL

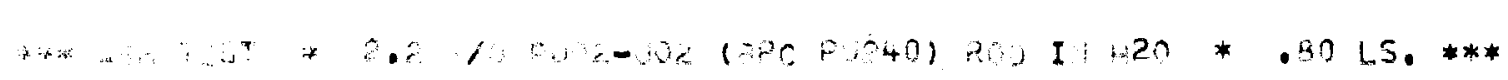

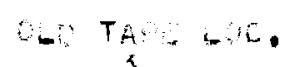

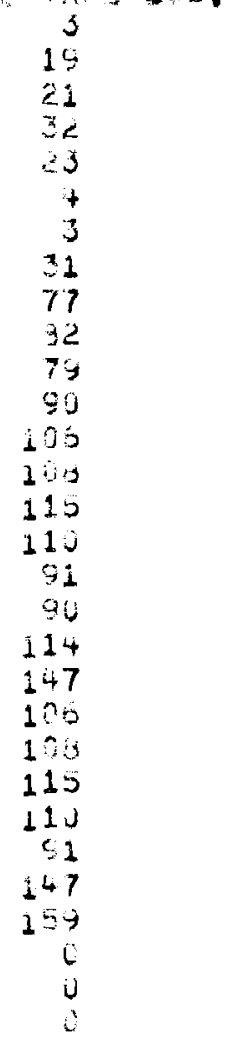

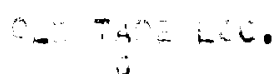

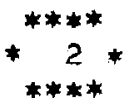

NO. ENFRGY POINTS

116
116

116

116

115

116

116

116

116

116

116

116

116

116

116

116

116

116

115

116

116

116

116

116

116

116

116

116

116

116

D. ENERGY POINTS 


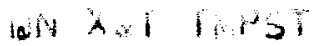




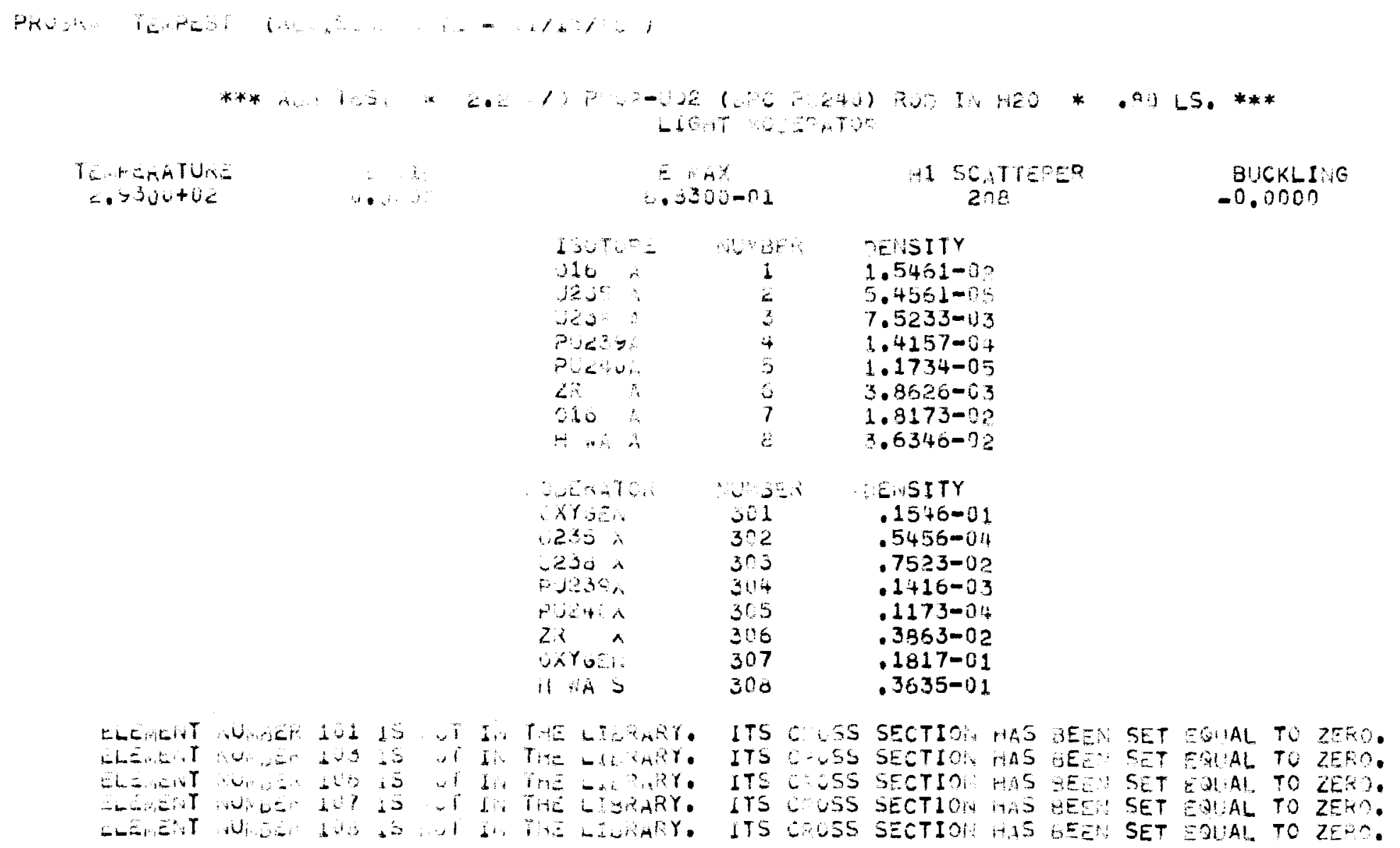




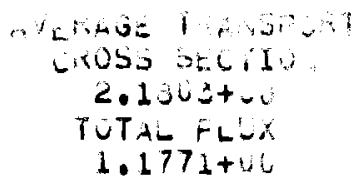

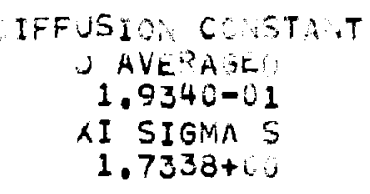

\begin{tabular}{|c|c|c|}
\hline LLCOU & $I_{L} \quad U_{B} \mathrm{EEF}$ & ICNO CROSS SECTION \\
\hline $2+0$ A & 1 & $1.2595-04$ \\
\hline $\sin A$ & 2 & $4.126 n+92$ \\
\hline$\angle C D A$ & 3 & $1.7286+00$ \\
\hline$p: j \leq 3 y A$ & 4 & $8.2628+02$ \\
\hline$r U_{c}+J A$ & 5 & $1.9614+02$ \\
\hline$\angle \therefore \quad A$ & 0 & $1.3063=01$ \\
\hline 18 & 7 & $1.8173-04$ \\
\hline 1: $4 \mathrm{~A}$ & s & $2.9991-01$ \\
\hline $2 \pi 35 \mathrm{~F}$ & $\$ 02$ & $3.5233+02$ \\
\hline$\because j \in J \ni F$ & 104 & $5.5431+02$ \\
\hline$P L, 40 F$ & 105 & $3.0782-02$ \\
\hline$u d t \quad x$ & 201 & $3.3342+00$ \\
\hline $\cos x$ & 202 & $8.2609+00$ \\
\hline $2<38 x$ & 203 & $6.6831+00$ \\
\hline Pن239x & 204 & $7.9305+00$ \\
\hline pist & 205 & $7.9305+00$ \\
\hline $6 k$ & 206 & $5.8804+00$ \\
\hline$\therefore b$ & 207 & $4.5290+00$ \\
\hline$\therefore x$ & 208 & $3.5947+01$ \\
\hline$\therefore: 021$ & 301 & $3.7776-01$ \\
\hline $205 x$ & 302 & $7.049 g=02$ \\
\hline$-20.35 x$ & 503 & $5.6295-02$ \\
\hline$f(3)=$ & 304 & $6.6565-02$ \\
\hline$+\cos +u$ & 305 & $6.6247-02$ \\
\hline$\therefore$ & 3110 & $1.2935-01$ \\
\hline वine & 507 & $3.1311-.71$ \\
\hline$\therefore$ i 5 & 213 & $4.7261+01$ \\
\hline$F$ Pun & 901 & $8.25+7-01$ \\
\hline 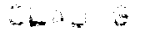 & 302 & $9.4033-11$ \\
\hline$\therefore$ & 903 & $1.1255+00$ \\
\hline
\end{tabular}




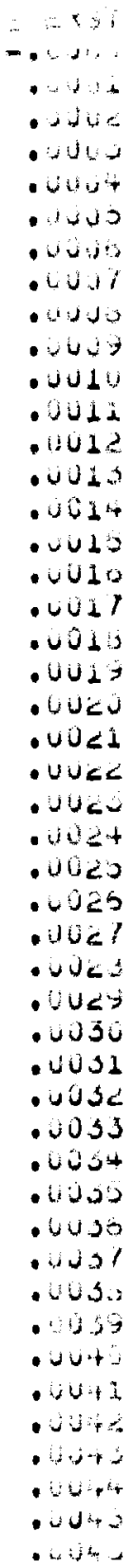

$\therefore 3$

? a 2

$\therefore 9000+4$

$4.1310+$

$5.53 .247+6$

$6.10 .5521+$

2.727340+6

$7.2375 .7+62$

$7.756750+0$

$. i+1314+i$

$\therefore 46,6=0+1$

- $30+22+$

$90190120+$

- $2159+\ldots$

$3 .+65=3+w$

$1.3+107 \leq+1$

i. $\Leftrightarrow 257 c+c$

. $109631+$

1... $525: 5+6$

1. 1. 1069t?

1. $1+207+1$

$1.104950+41$

$1.136890+61$

1. $-773-2+u 1$

1. $\operatorname{coc} s+c+u$

1. 21+ojoot

2. $207 J i v+w 1$

$1.205433+i$

1. $23234+41$

1. $205+1+4$

1. $357510+1$

acos $20 y+u$

1. $35=10-1.1$

$1.30427 i+01$

1. $595,915+01$

1. $+13137+21$

1. $+25 s 1+4+1$

$1.40301+1$

$\therefore 5+405+1$

. . - $0.25+1$

$1 .+7 \times 447+61$

i. $330+i$

$\therefore$ 1.03eta

10.130tit

1. $-2+3+4+2$

1. . . $130+6$

i. $321+4$
$D, 7(\cdots)$

$-1.0050$

1. $3021-1=$

- 110

$6.175722-01$

$7.689214-21$

$9.190550-01$

$1.057972+00$

$1.215736+00$

1. $362274+00$

1. $+92241+00$

$1.6345+1+30$

$1.7755+7+00$

$1.915232+00$

2. $053553+0 n$

2. $190470+10$

2. $3250-5+00$

$2.459075+0$

$2.591704+00$

$2.721513+10$

$2.3459 .5+00$

$2.977511+00$

$3.104392+00$

$3.231114+00$

$3.356216+00$

$3.40 \hat{2}+0+0 ?$

$3.503<73+00$

$3.7252+1+00$

3. $346311+0$ ?

3. $966.403+00$

4. $085232+0$

4. $203323+0$

$4.319710+30$

$4.435072+00$

$4.54940 y+00$

$4.50 ? 760+00$

$+.775026+0$

$+.386459+3$

$4.99691+2$

$5.1464+10$

$5.215651+0$

$5.325017+$

$0 .+27352+$

$5.534670+1$

$5.539301+5$

$5.743620+$

$5.846109+$
$=2 i(E)$

- onnos

$1.562406-05$

$6.224896-05$

1.395065-14

2. 470313-04

$3.944607-04$

$5.514341-04$

$7.475947-04$

$9.725887-04$

1. $226065-03$

1. $492240-03$

1.797995-03

2. $130656-n 3$

2.489801-03

$2.874901-0 x$

3. $235705-n 3$

$3.721422-0 \pi$

$4.181491=03$

$4.665057-03$

$5.171076-03$

5.697871-nर

$6.252773-23$

$6.930762-03$

7.4315s1-03

5. $054913-73$

6.700601-13

3. 3624 $37-03$

1. $005815-92$

1.076957-??

1. $1502: 3-02$

1.225750-0?

1. $303024-1 ?$

1. $333307-02$

$1.463574-1$ ?

$1.545799-02$

i. $531959-02$

1.719131-72.

1. $307994-12$

1. 393326-?

$1.901507-10$

$2.085020-7 ?$

2. 1922.32-12

2.2ค0201-?

$3.379976-32$

2. $41332-7 ?$

2. $54453-2 ?$

$2.569252-0$ 

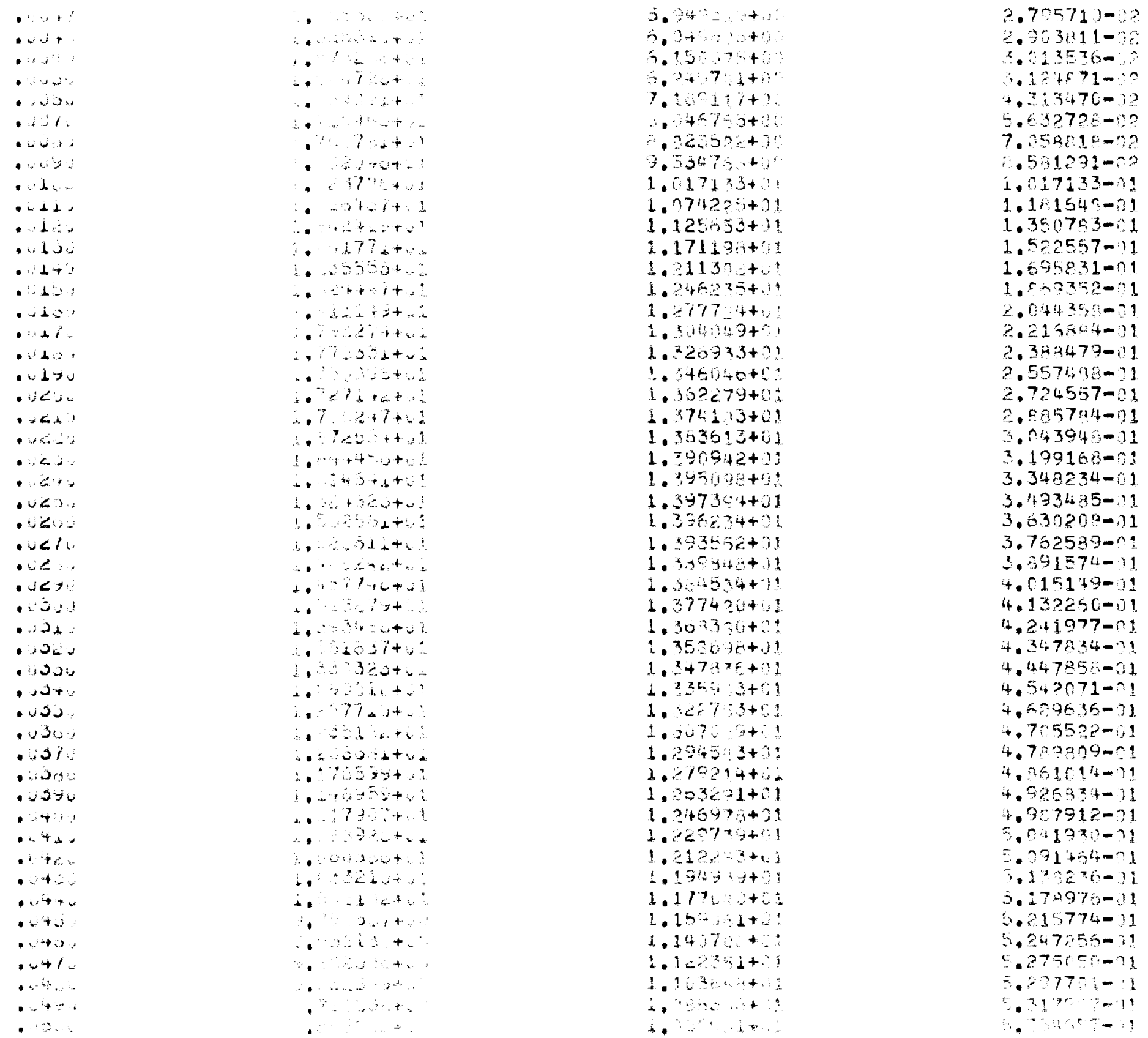
1. 354820-?1

1. $253990-01$ 1. $173702-1$

1. 10 1958-1 1. 259262-?1 1. $13677-.1$ $9.769211-92$ 9.465971-12 3. $203417-02$ $9.043373-02$ 8.925x00-72 $3.876751-12$ 8. $8736 \times 2-02$ $9.099717-02$ . $979632-12$ $0.979632-12$
$9.116975-12$ 5.316453-0? $9.523994-02$ $0.731400-02$ $9.731400=02$
$3.956979-02$ 1. $015191-01$ 1. $0364: 60-01$ 1.055100-01 $1.0724+1-01$ 1. $0390+3-11$ 1. $101333-31$ $1.113064-91$ 1. 121677-01 $1.12902 a-01$ 1.135251-71 $1.142757-01$ 1. $147+71-31$ 1.1.52106-71 $1.15511^{3}-01$ $1.160750-9$

$1.16+270-11$ 1. $16<6 \cdot 1-01$ 
$\therefore 175-10$

$\therefore 501-6$

$\therefore \operatorname{sel} 10-02$

$1.770480-62$

t. 50900-06

$+.551691-i 2$

$+.444833-12$

. $34+1393-46$

$+0+2364-42$

$+.15050-\mathrm{da}$

155212-i

$\therefore 605992-v 2$

. 9005i-u?

$3.7 \times 7) 25-0$

$6.717 .29-4$

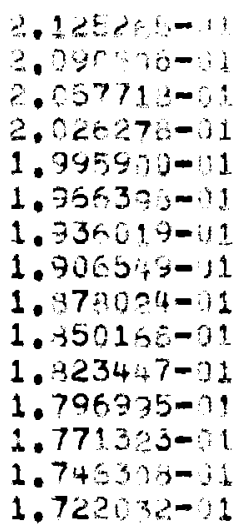

1. $36099-11$

1. $9065+s=11$

$1.979024-1$

1. $-350158-01$

$1.323447-01$

1.796935-11

1.77130j-1

$1.746370-3$

1. $72 ? 02-1$

$1.164 \times 94-11$

$1.170051-1$

1.17289?-31

1. $1752 \div+1-1$

$1.1775 a 1-0$

1.179839-71

1.180972-01

1. $132050-01$

1. 193155-01

1. $184103-? 1$

$1.185241-11$

1.196016-n1

1.135796-?

1. $187430-01$

1. $183202-2$ 


\section{REFERENYES}

1. Amcuyal, A. and P. Eenoist. New Method for Determining the Thermal Utilization Factor of a Cel1. CEA-572. February 16, 1960 .

2. Amoliyal, A., P. Beneist and J. Korowtz. Nouvelle Method de Determinatiof du Facteur $D^{i}$ utiliation Thermique $D^{i}$ une Ceilule. J. Nuclear Energy, 1957, Fin. 6, pp 79 to 98. Pergamon press Lta. Iondon.

3. Shudde, R.H. and $\because$ Dyen. THMPES, A Neutron Thermalization Code.

4. Strawhidge, I. E。 ard R. E. Barry. Giticality Calculations for Uniform Water-Moderated Lattices. Mivelear Scienee and Engineering: 23, 58-73 (1965).

5. IEM Systems Reference Iibrary Maruai。 IFM Form Mo. C28-6274-2.

6. Tomorito, ت. R. awd D. Petman. Private Comminication.

T. Fried, B. Private Oomurication。

8. Abramowitz: Mo and Irens A, Stegun (Eds.). Handbook of Mathematica) Functions. (Nationei Bureali of Standards, AMS 55) pp. 358 to 389.

9. Amster, Harvey ard Rolant Suarez. The Calculation of the rmal Constente Averaged over a Wigner-Wilkins Fux Spectrum. WAPD-TM-39. January $205 \%$ 


\section{DISTRIBUTION}

Number of Copies

1

3

$7 \quad$ Douglas United Nuclear

E. E. Mills

W. S. Nechodom

G. F. Owsley

Hans Toffer

F. Bouse (3)

2

Isochem

$$
\text { R. D. Carter W. P. McCue }
$$

1- University of Washington

Department of Nuclear Engineering

A. L. Babb

1 Westinghouse Electric Corporation - Pennsylvania Nuclear Development

I. E, Strawbridge 
S. J. Altschuler

J. L. Baker

C. L. Bennett

R. A. Bennett

S. R. Bierman

C. L. Brown

W. I. Bunch

G. J. Busselman

J. L. Carter

D. E. Christensen

R. G. Clark

E. D. Clayton

G. M. Dalen

E. C. Davis, Jr.

F. G. Dawson

D. E. Deonigi

B. H. Duane

S. L. Engstrom

D. G. Foster

H. A. Fowler

H. C. FOX

A. G. Gibbs

D. W. Glasgow

C. E. Haines

L. E. Hansen

G. E. Hanson

R. W. Hardie

O. K. Harling

R. A. Harris

C. M. Heeb

R. E. Heineman

H. L. Henry

G. M. Hess III

N. A. Hill

P. L. Hofmann

R. H. Holeman

J. G. Holt

U. P. Jenquin

G. J. Konzek

D. A. Kottwitz

J. W. Kutcher

D. D. Lanning

J. H. Lauby

R. C. Liikala

D. L, Lessor

C. W. Lindenmeier
W. W. Little, Jr.

R. C. Iloya

L. I. Meas

C. A. Mansius

D. R. Marr

R. P. Matsen

D. D. Matsumoto

E. T. Merrill

D. F. Newman

T. J. Oakes

L. J. Page

W. W. Porath

W. I. Purcell

W. C. Roberts

R. E. Schenter

I. C. Schmid

J. R. Sheff

D. R. Skeen (10)

R. I. Smith

W. P. Stinson

D. H. Thomsen

V. 0. Uotinen

A. D. Vaughn

A. E. Waltar

I. D. Williams

J. R. Worden

W. R. Young

H. S. Zwibel

Technical Information Files (5)

Technical Publications (2) 